\title{
Betting on the Real Line
}

\section{Citation}

Gao, Xi, Yiling Chen, and David M. Pennock. 2009. Betting on the real line. In Internet and Network Economics, 5th International Workshop, WINE 2009, Rome, Italy, December 2009, Proceedings, ed. Stefano Leonardi, 553-560. Berlin/Heidelberg: Springer-Verlag.

\section{Published Version}

doi:10.1007/978-3-642-10841-9_55

\section{Permanent link}

http://nrs.harvard.edu/urn-3:HUL.InstRepos:9943223

\section{Terms of Use}

This article was downloaded from Harvard University's DASH repository, and is made available under the terms and conditions applicable to Open Access Policy Articles, as set forth at http:// nrs.harvard.edu/urn-3:HUL.InstRepos:dash.current.terms-of-use\#OAP

\section{Share Your Story}

The Harvard community has made this article openly available.

Please share how this access benefits you. Submit a story.

Accessibility 


\title{
Betting on the Real Line
}

\author{
Xi Gao ${ }^{1}$, Yiling Chen ${ }^{1, \star}$, and David M. Pennock ${ }^{2}$ \\ 1 Harvard University, \{xagao, yiling\}@eecs .harvard.edu \\ 2 Yahoo! Research, pennockd@yahoo-inc.com
}

\begin{abstract}
We study the problem of designing prediction markets for random variables with continuous or countably infinite outcomes on the real line. Our interval betting languages allow traders to bet on any interval of their choice. Both the call market mechanism and two automated market maker mechanisms, logarithmic market scoring rule (LMSR) and dynamic parimutuel markets (DPM), are generalized to handle interval bets on continuous or countably infinite outcomes. We examine problems associated with operating these markets. We show that the auctioneer's order matching problem for interval bets can be solved in polynomial time for call markets. DPM can be generalized to deal with interval bets on both countably infinite and continuous outcomes and remains to have bounded loss. However, in a continuous-outcome DPM, a trader may incur loss even if the true outcome is within her betting interval. The LMSR market maker suffers from unbounded loss for both countably infinite and continuous outcomes.
\end{abstract}

Key words: Prediction Markets, Combinatorial Prediction Markets, Expressive Betting

\section{Introduction}

Prediction markets are speculative markets created for forecasting random variables. In practice, they have been shown to provide remarkably accurate probabilistic forecasts $[1,2]$. Existing prediction markets mainly focus on providing an aggregated probability mass function for a random variable with finite outcomes or discretized to have finite outcomes. For example, to predict the future printer sales level, the value of which lies on the positive real line, Hewlett-Packard's sales prediction markets partition the range of the sales level into about 10 exclusive intervals, each having an assigned Arrow-Debreu security that pays off $\$ 1$ if and only if the future sales level falls into the corresponding interval [3]. The price of each security represents the market probability that the sales level is within the corresponding interval. The set of prices provides a probability mass function for the discretized random variable.

However, many random variables of interest have continuous or countably infinite outcome spaces. For example, the carbon dioxide emission level in a certain period of time can be thought of as a continuous random variable on the positive real line; the printer sales level can be treated as a random variable with

* Part of this work was done while Yiling Chen was at Yahoo! Research. 
countably infinite outcomes, taking positive integer values. Discretizing such random variables into finite outcomes can potentially hurt information aggregation, as market participants may have information that can not be easily expressed with the ex-ante specified discretization. It is desirable to provide more expressive betting languages so that market participants can express their information more accurately and preferably in the same way they possess it.

In this paper, we design and study prediction market mechanisms for predicting random variables with continuous or countably infinite outcomes on the real line. We provide betting languages that allow market participants to bet on any interval of their choice and create the security on the fly. We generalize both the call market mechanism and two automated market maker mechanisms, logarithmic market scoring rules (LMSR) [4] and dynamic parimutuel markets (DPM) $[5,6]$, to handle interval bets on continuous or countably infinite outcomes, and examine problems associated with operating these markets. We show that the auctioneer's order matching problem can be solved in polynomial time for call markets. DPM can be generalized to deal with interval bets on both countably infinite and continuous outcomes and remains to have bounded loss. However, in a continuous-outcome DPM, a trader may incur loss even if the interval she bets on includes the true outcome. The LMSR market maker suffers from unbounded loss for both countably infinite and continuous outcomes. Due to space constraints, the Appendix is omitted and available upon request.

Related Work. Our work is situated in the broad framework of designing combinatorial prediction market mechanisms that provide more expressiveness to market participants. Various betting languages for permutation combinatorics have been studied for call markets, including subset betting and pair betting [7], singleton betting [8], and proportional betting [9]. Fortnow et al. [10] analyzed betting on Boolean combinatorics in call markets. For LMSR market makers, Chen et al. [11] showed that computing the contract price is \#P-hard for subset betting, pair betting, and Boolean formulas of two events. In a tournament setting, pricing in LMSR becomes tractable for some restricted Boolean betting languages [12]. Yoopick is a combinatorial prediction market implementation of LMSR that allows traders to bet on point spreads of their choice for sporting events [13]. It is implemented as a LMSR with a large number of finite outcomes. Agrawal et al. [14] proposed Quad-SCPM, which is a market maker mechanism that has the same worst-case loss as a quadratic scoring rule market maker. Quad-SCPM may be used for interval bets on countably infinite outcomes since its worst-case loss does not increase with the size of the outcome space.

\section{Background}

In this section, we briefly introduce three market mechanisms that have been used by prediction markets to predict random variables with finite outcomes.

\subsection{Call Markets}

A call market is an auctioneer mechanism, where the auctioneer (market institution) risklessly matches received orders. In a call market, participants submit 
buy or sell orders for individual contracts. All orders are assembled at one time in order to determine a market clearing price at which demand equals supply. Buy orders whose bid prices are higher than the clearing price and sell orders whose ask prices are lower than the clearing price are accepted. All transactions occur at the market clearing price. Most call markets are bilateral - matching buy and sell orders of the same contract. For multi-outcome events, call markets can be multilateral - allowing participants to submit orders on different contracts and performing global order matching [15-18].

\subsection{Logarithmic Market Scoring Rules}

Let $v$ be a random variable with $N$ mutually exclusive and exhaustive outcomes. A logarithmic market scoring rule (LMSR) $[4,19]$ is an automated market maker that subsidizes trading to predict the likelihood of each outcome. An LMSR market maker offers $n$ contracts, each corresponding to one outcome and paying $\$ 1$ if the outcome is realized $[4,20]$. Let $q_{i}$ be the total quantity of contract $i$ held by all traders combined, and let $\mathbf{q}$ be the vector of all quantities held. The market maker utilizes a cost function $C(\mathbf{q})=b \log \sum_{j=1}^{N} e^{q_{j} / b}$ that records the total amount of money traders have spent. A trader that wants to buy any bundle of contracts such that the total number of outstanding shares changes from $\mathbf{q}$ to $\tilde{\mathbf{q}}$ must pay $C(\tilde{\mathbf{q}})-C(\mathbf{q})$ dollars. Negative quantities encode sell orders and negative "payments" encode sale proceeds earned by the trader. At any time, the instantaneous price of contract $i$ is $p_{i}(\mathbf{q})=\frac{e^{q_{i} / b}}{\sum_{j=1}^{N} e^{q_{j} / b}}$, representing the cost per share of purchasing an infinitesimal quantity. An LMSR is built upon the logarithmic scoring rule, $s_{i}(\mathbf{r})=b \log \left(r_{i}\right)$. It is known that if the market maker starts the market with a uniform distribution its worst-case loss is bounded by $b \log N$.

\subsection{Dynamic Parimutuel Markets}

A dynamic parimutuel market (DPM) $[5,6]$ is a dynamic-cost variant of a parimutuel market. From a trader's perspective, DPM acts as a market maker in a similar way to LMSR. There are $N$ securities offered in the market, each corresponding to an outcome of the random variable. The cost function of the market maker, which captures the total money wagered in the market, is $C(\mathbf{q})=$

$\kappa \sqrt{\sum_{j=1}^{N} q_{j}^{2}}$, while the instantaneous price for contract $i$ is $p_{i}(\mathbf{q})=\frac{\kappa q_{i}}{\sqrt{\sum_{j=1}^{N} q_{j}^{2}}}$, where $\kappa$ is a free parameter. Unlike in LMSR, the contract payoff in DPM is not a fixed $\$ 1$. If outcome $i$ happens and the quantity vector at the end of the market is $\mathbf{q}^{f}$, the payoff per share of the winning security is $o_{i}=\frac{C\left(\mathbf{q}^{f}\right)}{q_{i}^{f}}=\frac{\kappa \sqrt{\sum_{j}\left(q_{j}^{f}\right)^{2}}}{q_{i}^{f}}$.

A nice property of DPM is that if a trader wagers on the correct outcome, she is guaranteed to have non-negative profit, because $p_{i}$ is always less than or equal to $\kappa$ and $o_{i}$ is always greater than or equal to $\kappa$. Because the price functions are not well-defined when $\mathbf{q}=\mathbf{0}$, the market maker must begin with a non-zero quantity vector $\mathbf{q}^{0}$. Hence, the market maker's loss is bounded by $C\left(\mathbf{q}^{0}\right)$. 


\section{Call Markets for Interval Betting}

For a random variable $X$ that has continuous or countably infinite outcomes on the real line, we consider the betting language that allows traders to bet on any interval $(l, u)$ of their choice on the real line and create a security for the interval on the fly. The security pays off $\$ 1$ per share when the betting interval contains the realized value of $X$. For countably infinite outcomes, the interval is interpreted as a set of outcomes that lie within the interval.

Suppose that the range of $X$ is (L, U) where $L \in \Re \cup\{-\infty\}$ and $U \in \Re \cup$ $\{+\infty\}$. Traders submit buy orders. Each order $i \in O$ is defined by $\left(b_{i}, q_{i}, l_{i}, u_{i}\right)$, where $b_{i}$ denotes the bid price for a unit share of the security on interval $\left(l_{i}, u_{i}\right)$, and $q_{i}$ denotes the number of shares of the security to purchase at price $b_{i}$. We note $l_{i} \geq L$ and $u_{i} \leq U$. Given a set of orders $O$ submitted to the auctioneer, the auctioneer needs to decide which orders can be risklessly accepted. We consider the auctioneer's problem of finding an optimal match to maximize its worst-case profit given a set of orders $O$.

We first define the state space $S$ to be the partition of the range of $X$ formed by orders $O$. For any order $i \in O,\left(l_{i}, u_{i}\right)$ defines 2 boundary points of the partition. Let $A=\left(\cup_{i \in O} l_{i}\right) \cup\{L\}$ be the set of left ends of all intervals in $O$ and the left end of the range of $X$, and $B=\left(\cup_{i \in O} u_{i}\right) \cup\{U\}$ be the set of right ends of all intervals in $O$ and the right end of the range of $X$. We rank all elements of $A$ and $B$ in order of increasing values, and denote the $i$-th element as $e_{i}$. Clearly, $e_{1}=L$ and $e_{|A|+|B|}=U$. We formally define the state space $S$ as follows.

Definition 1. Let $s_{i} \in S$ be the $i$-th element of the state space $S$ for all $1 \leq$ $i \leq(|A|+|B|-1)$. If $e_{i}=e_{i+1}$, then $s_{i}=\left\{e_{i}\right\}$. Otherwise, $s_{i}=\left(e_{i}, e_{i+1}\right]$ if both $e_{i} \in A$ and $e_{i+1} \in A ; s_{i}=\left(e_{i}, e_{i+1}\right)$ if $e_{i} \in A$ and $e_{i+1} \in B ; s_{i}=\left[e_{i}, e_{i+1}\right]$ if $e_{i} \in B$ and $e_{i+1} \in A$; and $s_{i}=\left[e_{i}, e_{i+1}\right)$ if $e_{i} \in B$ and $e_{i+1} \in B$.

Because $|S|=|A|+|B|-1,|A| \leq|O|+1$, and $|B| \leq|O|+1$, we have $|S| \leq 2|O|+1$.

With the definition of states given orders $O$, we formulate the auctioneer's optimal match problem as a linear program, analogous to the one used for permutation betting [7].

Definition 2 (Optimal Match). Given a set of order $O$, choose $x_{i} \in[0,1]$ such that the following linear program is optimized.

$$
\begin{array}{cc}
\max _{x_{i}, c} & c \\
\text { s.t. } \sum_{i}\left(b_{i}-I_{i}(s)\right) q_{i} x_{i} \geq c, & \forall s \in S \\
0 \leq x_{i} \leq 1, \quad \forall i \in O
\end{array}
$$

$I_{i}(s)$ is the indicator variable for whether order $i$ is winning in state $s . I_{i}(s)=1$ if the order gets a payoff of $\$ 1$ in $s$ and $I_{i}(s)=0$ otherwise. The variable $c$ represents the worst-case profit for the auctioneer, and $x_{i} \in[0,1]$ represents the fraction of order $i \in O$ that is accepted. As the number of structural constraints is at most $2|O|+1$ and the number of variables is $|O|$, (1) can be solved efficiently. We state it in the following theorem. 
Theorem 3. For call markets, the auctioneer's optimal order matching problem for interval betting on countably infinite and continuous outcomes can be solved in polynomial time.

Thus, if the optimal solution to (1) generates positive worst-case profit $c$, the auctioneer accepts orders according to the solution. Otherwise, when $c \leq 0$, the auctioneer rejects all orders.

When there are few traders in the market, finding a counterpart to trade in a call market may be hard and the market may suffer from the thin market problem. Allowing traders to bet on different intervals further exacerbates the problem by dividing traders' attention among a large number of subsets of securities, making the likelihood of finding a multi-lateral match even more remote. In addition, call markets are zero-sum games and hence are challenged by the no-trade theorem [21]. In the next two sections, we examine market maker mechanisms, which not only provide infinite liquidity but also subsidize trading, for interval betting.

\section{Dynamic Parimutuel Markets for Interval Betting}

For interval betting in DPMs, traders also create a security on the fly by choosing an interval $(l, u)$. However, the payoff of the security is not fixed to be $\$ 1$. Instead, each share of the security whose interval contains the realized value of the random variable entitles its holder to an equal share of the total money in the market. We generalize DPM to allow for (but not limited to) interval betting on countably infinite and continuous outcomes. The problem that we consider is whether these mechanisms still ensure the bounded loss of the market maker.

\subsection{Infinite-Outcome DPM}

We generalize DPM to allow for countably infinite outcomes, and call the resulting mechanism infinite-outcome DPM. In an infinite-outcome DPM, the underlying forecast variable can have countably infinite mutually exclusive and exhaustive outcomes. Each state security corresponds to one potential outcome. An interval bet often includes a set of state securities. The market maker keeps track of the quantity vector of outstanding state securities, still denoted as $\mathbf{q}$, which is a vector of dimension $\infty$. The cost and price functions for the infiniteoutcome DPM are $C^{I}(\mathbf{q})=\kappa \sqrt{\sum_{j=1}^{\infty} q_{j}^{2}}$, and $p_{i}^{I}(\mathbf{q})=\frac{\kappa q_{i}}{\sqrt{\sum_{j=1}^{\infty} q_{j}^{2}}}$. The payoff per winning security if outcome $i$ happens is $o_{i}^{I}=\frac{\kappa \sqrt{\sum_{j=1}^{\infty}\left(q_{j}^{f}\right)^{2}}}{q_{i}^{f}}$.

The loss of the market maker in an infinite-outcome DPM is still her cost to initiate the market. The market maker needs to choose an initial quantity vector $\mathbf{q}^{0}$ such that her loss $C^{I}\left(\mathbf{q}^{0}\right)$ is finite. In practice, an infinite-outcome DPM market maker can start with a quantity vector that has only finite positive elements and all others are zeros, or use an infinite converging series. Whenever a trader purchases a state security whose current price is zero or that has not 
been purchased before, the market maker begins to track quantity and calculate price for that security. Hence, infinite-outcome DPM can be operated as a finiteoutcome DPM that can add new state securities as needed. The market maker does not need to record quantities and calculate prices for all infinite outcomes, but only for those having outstanding shares. Infinite-outcome DPM maintains the desirable price-payoff relationship of DPM - the payoff of a security is always greater than or equal to $\kappa$ and its price is always less than or equal to $\kappa$.

\subsection{Continuous-Outcome DPM}

We then generalize DPM to allow for continuous outcomes, and call the resulting mechanism continuous-outcome DPM. The cost and price functions of a continuous-outcome DPM are $C=\kappa \sqrt{\int_{-\infty}^{+\infty} q(y)^{2} d y}$ and $p(x)=\frac{\kappa q(x)}{\sqrt{\int_{-\infty}^{+\infty} q(y)^{2} d y}}$. A trader can buy $\delta$ shares of an interval $(l, u)$. The market maker then increases $q(x)$ by $\delta$ for all $x \in(l, u)$. The trader's payment equals the change in value of the cost function. However, strictly speaking, function $p(x)$ does not represent price, but is better interpreted as a density function. The instantaneous price for buying infinitely small amounts of the security for interval $(l, u)$ is $p_{(l, u)}=\int_{l}^{u} p(x) d x=\frac{\kappa \int_{l}^{u} q(x) d x}{\sqrt{\int_{-\infty}^{+\infty} q(y)^{2} d y}}$. If the realized value of the random variable is $x^{*}$, each share of a security on any interval that contains $x^{*}$ has payoff $o\left(x^{*}\right)=\frac{C}{q^{f}\left(x^{*}\right)}=\frac{\kappa \sqrt{\int_{-\infty}^{+\infty} q^{f}(y)^{2} d y}}{q^{f}\left(x^{*}\right)}$, where $q^{f}(y)$ is the number of outstanding shares for securities whose interval contains $y$ at the close of the market.

A continuous-outcome DPM market maker can choose an initial quantity distribution $q^{0}(x)$ such that her loss is finite. However, the desirable price-payoff relationship that holds for the original DPM no longer holds for continuousoutcome DPM. A trader who bets on the correct outcome may still lose money. Theorem 4 states the price-payoff relationship for continuous-outcome DPM. Proof of the theorem is provided in Appendix A.

Theorem 4. The price per share for buying a security on interval $(l, u)$ is always less than or equal to $\kappa \sqrt{u-l}$. If traders can bet on any non-empty open interval, the payoff per share is bounded below by 0. If traders could bet only on open intervals of size at least $z$, the payoff per share is bounded below by $\frac{\kappa \sqrt{2 z}}{2}$.

\section{Logarithmic Market Scoring Rule for Interval Betting}

For LMSR, we define the same interval betting language as in call markets. A trader can create a security by specifying an interval $(l, u)$ to bet on. If the realized value of $X$ falls into the interval, the security pays off $\$ 1$ per share. We generalize LMSR to allow countably infinite and continuous outcomes and study whether the market maker still has bounded loss.

LMSR for finite outcomes can be extended to accommodate interval betting on countably infinite outcomes simply by changing the summations in the price 
and cost functions to include all countably infinite outcomes. However, as the LMSR market maker's worst-case loss is $b \log N$, the market maker's worst-case loss is unbounded as $N$ approaches $\infty$.

We generalize LMSR to accommodate continuous outcome spaces. A logarithmic scoring rule for a continuous random variable is $s(r(x))=b \log (r(x))$ where $x$ is the realized value for the random variable and $r(x)$ is the reported probability density function for the random variable evaluated at $x$. Using an equation system similar to the one proposed by Chen and Pennock [20], we derive the corresponding price and cost functions for the continuous logarithmic

scoring rule: $C=b \log \left(\int_{-\infty}^{+\infty} e^{q(y) / b} d y\right)$, and $p(x)=\frac{e^{q(x) / b}}{\int_{-\infty}^{+\infty} e^{q(y) / b} d y}$. Here, $p(x)$ does not represent price, but is best interpreted as a density function. The instantaneous price for buying infinitely small amounts of the security for interval $(l, u)$ is $\int_{l}^{u} p(x) d x$. If the interval $(l, u)$ contains the realized value, one share of the security entitles its holder $\$ 1$ payoff.

However, the worst-case loss is still unbounded for a continuous LMSR market maker even with the restriction on the size of allowable intervals, as shown by Theorem 5. Proof of the theorem is presented in Appendix B.

Theorem 5. A continuous logarithmic market scoring rule market maker has unbounded worst-case loss, with or without the restriction that traders can bet only on intervals of size at least $z$.

\section{Conclusion and Future Directions}

We study interval betting on random variables with continuous or countably infinite outcomes for call markets, DPM, and LMSR. We show that the auctioneer's order matching problem in call markets can be solved in polynomial time for interval bets. DPM can be generalized to handle both countably infinite and continuous outcomes. Unfortunately, in a continuous-outcome DPM, a trader may incur loss even if her betting interval contains the true outcome. LMSR market makers, however, suffer from unbounded loss for both countably infinite and continuous outcomes.

One important future direction is to design automated market maker mechanisms with desirable properties, especially bounded loss, when handling continuous outcome spaces. In particular, it may be fruitful to explore interval bets with variable payoffs for outcomes within the interval. The interval contracts for call markets and LMSR give the same payoff as long as the outcome falls within the specified interval. Implicitly, this assumes that a trader's prediction of the random variable is a uniform distribution over the given interval. Alternatively, it would be interesting to allow for the trader's probability distribution of the random variable to take other shapes over the given interval, and hence to allow payoffs to vary correspondingly for outcomes within the interval.

\section{References}

1. Berg, J.E., Forsythe, R., Nelson, F.D., Rietz, T.A.: Results from a dozen years of election futures markets research. In Plott, C.A., Smith, V., eds.: Handbook of Experimental Economic Results. (2001) 
2. Wolfers, J., Zitzewitz, E.: Prediction markets. Journal of Economic Perspective 18(2) (2004) 107-126

3. Chen, K.Y., Plott, C.R.: Information aggregation mechanisms: Concept, design and implementation for a sales forecasting problem. Working paper No. 1131, California Institute of Technology (2002)

4. Hanson, R.D.: Combinatorial information market design. Information Systems Frontiers 5(1) (2003) 107-119

5. Pennock, D.M.: A dynamic pari-mutuel market for hedging, wagering, and information aggregation. In: ACM Conference on Electronic Commerce (EC). (2004)

6. Mangold, B., Dooley, M., Dornfest, R., Flake, G.W., Hoffman, H., Kasturi, T., Pennock, D.M.: The tech buzz game. IEEE Computer 38(7) (2005) 94-97

7. Chen, Y., Fortnow, L., Nikolova, E., Pennock, D.M.: Betting on permutations. In: ACM Conference on Electronic Commerce (EC). (2007) 326-335

8. Ghodsi, M., Mahini, H., Mirrokni, V.S., ZadiMoghaddam, M.: Permutation betting markets: singleton betting with extra information. In: ACM Conference on Electronic Commerce (EC). (2008) 180-189

9. Agrawal, S., Wang, Z., Ye, Y.: Parimutuel betting on permutations. In: Lecture Notes in Computer Science, The International Workshop on Internet and Network Economics (WINE). (2008)

10. Fortnow, L., Kilian, J., Pennock, D.M., Wellman, M.P.: Betting boolean-style: A framework for trading in securities based on logical formulas. Decision Support Systems 39(1) (2004) 87-104

11. Chen, Y., Fortnow, L., Lambert, N., Pennock, D.M., Wortman, J.: Complexity of combinatorial market makers. In: ACM Conference on Electronic Commerce (EC). (2008) 190-199

12. Chen, Y., Goel, S., Pennock, D.M.: Pricing combinatorial markets for tournaments. In: ACM Symposium on Theory of Computing (STOC). (2008) 305-314

13. Goel, S., Pennock, D., Reeves, D.M., Yu, C.: Yoopick: A combinatorial sports prediction market. In: AAAI. (2008) 1880-1881

14. Agrawal, S., Delage, E., Peters, M., Wang, Z., Ye, Y.: A unified framework for dynamic pari-mutuel information market design. In: ACM Conference on Electronic Commerce (EC). (2009) 255-264

15. Bossaerts, P., Fine, L., Ledyard, J.: Inducing liquidity in thin financial markets through combined-value trading mechanisms. European Economic Review (46) (2002) 1671-1695

16. Lange, J., Economides, N.: A parimutuel market microstructure for contingent claims trading. NYU School of Business Discussion Paper No. EC-01-13 (2007)

17. Baron, K., Lange, J.: Parimutuel Applications in Finance: New Markets for New Risks. Palgrave Macmillan (2005)

18. Peters, M., So, A.M.C., Ye, Y.: Pari-mutuel markets: Mechanisms and performance. In: Lecture Notes in Computer Science, The International Workshop on Internet and Network Economics (WINE). (2007) 82-95

19. Hanson, R.D.: Logarithmic market scoring rules for modular combinatorial information aggregation. Journal of Prediction Markets 1(1) (2007) 1-15

20. Chen, Y., Pennock, D.M.: A utility framework for bounded-loss market makers. In: Conference on Uncertainty in Artificial Intelligence (UAI 2007). (2007) 49-56

21. Milgrom, P., Stokey, N.L.: Information, trade and common knowledge. Journal of Economic Theory 26(1) (1982) 17-27 


\section{A Proof of Theorem 4}

Proof. By Cauchy-Schwarz inequality, we have

$$
\left(\int_{l}^{u} q(x) d x\right)^{2} \leq \int_{l}^{u} q(x)^{2} d x \int_{l}^{u} 1^{2} d x=(u-l) \int_{l}^{u} q(x)^{2} d x .
$$

Hence,

$$
\int_{l}^{u} q(x) d x \leq \sqrt{(u-l) \int_{l}^{u} q(x)^{2} d x} .
$$

The price of buying a security on interval $(l, u)$ is then

$$
p_{(l, u)}=\frac{\kappa \int_{l}^{u} q(x) d x}{\sqrt{\int_{-\infty}^{+\infty} q(y)^{2} d y}} \leq \frac{\kappa \int_{l}^{u} q(x) d x}{\sqrt{\int_{l}^{u} q(y)^{2} d y}} \leq \kappa \sqrt{u-l} .
$$

Suppose the smallest partition that includes the true outcome $x^{*}$ at the end of the market is $(c, d) \cdot q^{f}(x)=q^{f}\left(x^{*}\right)$ for all $x \in(c, d)$. Then, payoff per share is

$$
\begin{aligned}
o\left(x^{*}\right) & =\frac{\kappa \sqrt{\int_{-\infty}^{+\infty} q^{f}(y)^{2} d y}}{q^{f}\left(x^{*}\right)} \\
& \geq \frac{\kappa \sqrt{\int_{c}^{d} q^{f}(y)^{2} d y}}{q^{f}\left(x^{*}\right)} \\
& =\frac{\kappa \sqrt{q^{f}\left(x^{*}\right)^{2}(d-c)}}{q^{f}\left(x^{*}\right)} \\
& =\kappa \sqrt{d-c} .
\end{aligned}
$$

If traders can bet on any non-empty interval, $(d-c)$ in $(3)$ may approach 0 . Thus, payoff per share $o\left(x^{*}\right)>0$.

If the market maker restricts that traders can only bet on intervals no smaller than $z$, we consider the interval $(d-z, c+z)$ for the case of $(d-c)<z$. Because $(c, d)$ is the smallest partition that contains $x^{*}$,

$$
q^{f}\left(x^{*}\right)=\frac{\int_{c}^{d} q^{f}(x) d x}{d-c} .
$$

Any time when $\int_{c}^{d} q(x) d x$ is increased by $a$, it must be the case that $\int_{d-z}^{c} q(x) d x+\int_{d}^{c+z} q(x) d x$ is increased at least by $\frac{a}{d-c}(z-(d-c))$, because the smallest interval size is $z$. Hence,

$$
\int_{d-z}^{c} q^{f}(x) d x+\int_{d}^{c+z} q^{f}(x) d x \geq \frac{\int_{c}^{d} q^{f}(x) d x}{d-c}(z-(d-c)) .
$$


Thus,

$$
\begin{aligned}
\int_{d-z}^{c+z} q^{f}(x) d x & \geq \frac{\int_{c}^{d} q^{f}(x) d x}{d-c}(z-(d-c))+\int_{c}^{d} q^{f}(x) d x \\
& =\frac{z}{d-c} \int_{c}^{d} q^{f}(x) d x
\end{aligned}
$$

Payoff per share is

$$
\begin{aligned}
o\left(x^{*}\right) & =\frac{\kappa \sqrt{\int_{-\infty}^{+\infty} q^{f}(y)^{2} d y}}{q^{f}\left(x^{*}\right)} \\
& =\frac{\kappa(d-c) \sqrt{\int_{-\infty}^{+\infty} q^{f}(y)^{2} d y}}{\int_{c}^{d} q^{f}(x) d x} \\
& \geq \frac{\kappa(d-c) \sqrt{\int_{d-z}^{c+z} q^{f}(y)^{2} d y}}{\int_{c}^{d} q^{f}(x) d x} \\
& \geq \frac{\kappa(d-c) \int_{d-z}^{c+z} q^{f}(y) d y}{\sqrt{(c+z)-(d-z)} \int_{c}^{d} q^{f}(x) d x} \\
& \geq \frac{\kappa(d-c) \frac{z}{d-c} \int_{c}^{d} q^{f}(y) d y}{\sqrt{(c+z)-(d-z)} \int_{c}^{d} q^{f}(x) d x} \\
& =\frac{\kappa z}{\sqrt{2 z-(d-c)}} \\
& >\frac{\kappa \sqrt{2 z}}{2} .
\end{aligned}
$$

The second equality comes from (4). The fourth inequality is a result of applying (2). Applying (5), we get the fifth inequality. Letting $(d-c) \rightarrow 0$, we obtain the last inequality.

\section{B Proof of Theorem 5}

Proof. Let $x^{*}$ be the realized value of the random variable. Let $p^{0}\left(x^{*}\right)$ and $p^{f}\left(x^{*}\right)$ be the initial and final price density for $x^{*}$. Then, the market maker's loss is $b \log p^{f}\left(x^{*}\right)-b \log p^{0}\left(x^{*}\right)$. 
Suppose the smallest partition that includes the true outcome $x^{*}$ at the end of the market is $(c, d)$. If traders can bet on any non-empty intervals,

$$
\begin{aligned}
p^{f}\left(x^{*}\right) & =\frac{e^{q^{f}\left(x^{*}\right) / b}}{\int_{-\infty}^{+\infty} e^{q^{f}(y) / b} d y} \\
& \leq \frac{e^{q^{f}\left(x^{*}\right) / b}}{\int_{c}^{d} e^{q^{f}(y) / b} d y} \\
& =\frac{e^{q^{f}\left(x^{*}\right) / b}}{(d-c) e^{q^{f}\left(x^{*}\right) / b}}=\frac{1}{d-c} .
\end{aligned}
$$

As $(d-c)$ approaches $0, \frac{1}{d-c}$ approaches $\infty$. Hence, the worst-cast loss is not bounded because $b \log p^{f}\left(x^{*}\right)<\infty$.

If the smallest interval that traders can bet on is of size $z$, consider the situation that $(d-c)<z$ and traders buying equal shares of intervals $(d-z, d)$ and $(c, c+z)$. Then,

$$
\begin{aligned}
p^{f}\left(x^{*}\right) & =\frac{e^{q^{f}\left(x^{*}\right) / b}}{\int_{-\infty}^{+\infty} e^{q^{f}(y) / b} d y} \\
& \leq \frac{e^{q^{f}\left(x^{*}\right) / b}}{\int_{d-z}^{c} e^{q^{f}(y) / b} d y+\int_{c}^{d} e^{q^{f}(y) / b} d y+\int_{d}^{c+z} e^{q^{f}(y) / b} d y} \\
& =\frac{e^{q^{f}\left(x^{*}\right) / b}}{2(z-(d-c)) e^{q^{f}\left(x^{*}\right) / 2 b}+(d-c) e^{q^{f}\left(x^{*}\right) / b}} \\
& =\frac{1}{2(z-(d-c)) e^{-q^{f}\left(x^{*}\right) / 2 b}+(d-c)} .
\end{aligned}
$$

When $q^{f}\left(x^{*}\right) \rightarrow \infty$ and $(d-c) \rightarrow 0$, the $p^{f}\left(x^{*}\right)$ approaches to 0 according to the above expression. Hence, the worst-case loss of the market maker is not bounded because $b \log p^{f}\left(x^{*}\right)<\infty$. 